Bull. Korean Math. Soc. 48 (2011), No. 5, pp. 1023-1032

http://dx.doi.org/10.4134/BKMS.2011.48.5.1023

\title{
AN EKELAND TYPE VARIATIONAL PRINCIPLE ON GAUGE SPACES WITH APPLICATIONS TO FIXED POINT THEORY, DROP THEORY AND COERCIVITY
}

\author{
Jong-Sook Bae, Seong-Hoon Cho, And Jeong-Jin Kim
}

\begin{abstract}
In this paper, a new Ekeland type variational principle on gauge spaces is established. As applications, we give Caristi-Kirk type fixed point theorems on gauge spaces, and Daneš' drop theorem on seminormed spaces. Also, we show that the Palais-Smale condition implies coercivity on semi-normed spaces.
\end{abstract}

\section{Introduction}

The Ekeland's variational principle is one of the most important results in nonlinear analysis and very useful tools to solve problems in optimization, optimal control theory, game theory, nonlinear equations and dynamical systems $[2,3,4,13,17,18,24,34]$. Qiu [30] extends the result of Phelps [28, pages 47] to countable semi-normed spaces and obtain Ekeland's variational principle. Recently, the authors [2] gave an Ekeland type variational principle in quasi-metric spaces and a Caristi-Kirk type fixed point theorem for multivalued maps. Also, in [31, 32], the authors obtained Ekeland's variational principle and some related results in locally $p$-convex spaces.

We establish an Ekeland type variational principle for a countable family of lower semi-continuous functions defined on gauge spaces. This result is a generalization of Qiu's result [30].

In $[1,19,20,21]$, the authors obtained fixed point results on gauge spaces.

In Section 3, we give Caristi type fixed point theorems on gauge spaces.

We give applications of our results to drop properties and the Palais-Smale condition. Daneš' drop theorem [11] in Banach spaces is equivalent to the Ekeland's variational principle [27] and it was generalized to locally convex spaces by introducing the concept of a strong Minkowski separation of sets [10]. Zheng [36] extended this result to topological vector spaces. Qiu [29] generalized Daneš' drop theorem to locally convex spaces.

Received March 19, 2010; Revised June 11, 2010.

2010 Mathematics Subject Classification. 54H25, 49J40, 49J45.

Key words and phrases. variational principle, gauge space, fixed point, drop theorem, Palais-Smale condition. 
In Section 4, we generalize Daneš' drop theorem to a sequentially complete topological space whose topology is generated by a family of seminorms.

The Palais-Smale condition implies coercivity for a $C^{1}$ function which is bounded from below on a Banach space. In [8], the authors generalized this result under weaker regularity assumptions in a Banach space.

In Section 5, we generalize this result to a complete locally convex topological vector space generated by a family of seminorms.

\section{Variational theorems}

We denote by $\left(X,\left\{d_{\alpha}\right\}_{\alpha \in \Lambda}\right)$ the gauge space [14, pp. 198, 308] endowed with a gauge structure induced by a family $\left\{d_{\alpha}: \alpha \in \Lambda\right\}$ of pseudo metrics. Recall that a function $f: X \rightarrow \mathbb{R}$ is lower semi-continuous if, for any sequence $\left\{x_{n}\right\}$ in $X$ and $x \in X, g(x) \leq \underline{\lim }_{n \rightarrow \infty} g\left(x_{n}\right)$ whenever $\lim _{n \rightarrow \infty} x_{n}=x$.

We extend Ekeland's variational principle to gauge spaces.

Theorem 2.1. Let $\left(X,\left\{d_{\alpha}\right\}_{\alpha \in \Lambda}\right)$ be a complete gauge space, $\mathcal{F}$ be a countable family of lower semi-continuous functions from $X$ into $[0, \infty)$, and let $\phi: \Lambda \rightarrow$ $\mathcal{F}$ be a map defined by $\phi(\alpha)=\phi_{\alpha}$. Then for any $x_{0} \in X$, there exists $z \in X$ such that

(i) for each $\alpha \in \Lambda, d_{\alpha}\left(x_{0}, z\right) \leq \phi_{\alpha}\left(x_{0}\right)-\phi_{\alpha}(z)$,

(ii) for each $x \in X$ with $x \neq z$, there exists $\alpha \in \Lambda$ such that $d_{\alpha}(z, x)>$ $\phi_{\alpha}(z)-\phi_{\alpha}(x)$.

Proof. We define a relation $\leq$ on $X$ as follows.

For any $x, y \in X$,

$$
x \leq y \Longleftrightarrow d_{\alpha}(x, y) \leq \phi_{\alpha}(x)-\phi_{\alpha}(y) \text { for each } \alpha \in \Lambda .
$$

It is easy to show that $\leq$ is an ordering. For any $x \in X$, let $S(x)=\{y \in X$ : $x \leq y\}$, and $\mathcal{F}=\left\{\phi_{n}\right\}_{n=1}^{\infty}$. Define $\phi: X \rightarrow[0, \infty)$ by

$$
\phi(x)=\sum_{n=1}^{\infty} \frac{\phi_{n}(x)}{2^{n}\left(1+\phi_{n}(x)\right)} .
$$

Then, it is clear that $x \leq y$ implies $\phi(y) \leq \phi(x)$, and $x \leq y$ with $x \neq y$ implies $\phi(y)<\phi(x)$.

Let $x_{0} \in X$ be given. Take $x_{1} \in S\left(x_{0}\right)$ such that

$$
\phi\left(x_{1}\right)<\inf \left\{\phi(x): x \in S\left(x_{0}\right)\right\}+1 .
$$

Inductively, we can choose a sequence $\left\{x_{n}\right\}$ in $X$ such that

$$
x_{n+1} \in S\left(x_{n}\right) \text { and } \phi\left(x_{n+1}\right)<\inf \left\{\phi(x): x \in S\left(x_{n}\right)\right\}+\frac{1}{n+1} .
$$

Then, for any $\alpha \in \Lambda, d_{\alpha}\left(x_{n+1}, x_{n}\right) \leq \phi_{\alpha}\left(x_{n}\right)-\phi_{\alpha}\left(x_{n+1}\right)$ and so, for $m>n$,

$$
d_{\alpha}\left(x_{n}, x_{m}\right) \leq \phi_{\alpha}\left(x_{n}\right)-\phi_{\alpha}\left(x_{m}\right) \text {. }
$$


Since $\left\{\phi_{\alpha}\left(x_{n}\right)\right\}$ is nonincreasing, $\left\{x_{n}\right\}$ is a Cauchy sequence. By the completeness of $X, \lim _{n \rightarrow \infty} x_{n}=z \in X$ exists. Since each $\phi_{\alpha}$ is lower semicontinuous, $\lim \phi_{\alpha}\left(x_{n}\right) \geq \phi_{\alpha}(z)$ for each $\alpha \in \Lambda$.

Hence

$$
d_{\alpha}\left(x_{n}, z\right)=\lim _{m \rightarrow \infty} d_{\alpha}\left(x_{n}, x_{m}\right) \leq \phi_{\alpha}\left(x_{n}\right)-\phi_{\alpha}(z),
$$

and hence $z \in S\left(x_{n}\right)$ for each $n \in \mathbb{N}$.

If $x \in S(z)$, then $x \in S\left(x_{n}\right)$ for all $n \in \mathbb{N}$, and

$$
\inf \left\{\phi(x): x \in S\left(x_{n}\right)\right\} \leq \phi(x) \leq \phi(z) \leq \inf \left\{\phi(x): x \in S\left(x_{n}\right)\right\}+\frac{1}{n+1} .
$$

Since $n$ is an arbitrary natural number, we have $\phi(x)=\phi(z)$, and so $x=z$. Thus if $x \neq z$, then $x \notin S(z)$. Hence there exists $\alpha \in \Lambda$ such that $d_{\alpha}(z, x)>$ $\phi_{\alpha}(z)-\phi_{\alpha}(x)$. lary.

If $\mathcal{F}=\{\phi\}$ is a singleton in Theorem 2.1, then we have the following corol-

Collorary 2.2. Let $\left(X,\left\{d_{\alpha}\right\}_{\alpha \in \Lambda}\right)$ be a complete gauge space, and $\phi: X \rightarrow$ $[0, \infty)$ be a lower semi-continuous function. Then for any $x_{0} \in X$, there exists $z \in X$ such that

(i) $d_{\alpha}\left(x_{0}, z\right) \leq \phi\left(x_{0}\right)-\phi(z)$ for each $\alpha \in \Lambda$,

(ii) for each $x \in X$ with $x \neq z$, there exists $\alpha \in \Lambda$ such that $d_{\alpha}(x, z)>$ $\phi(z)-\phi(x)$.

By Corollary 2.2, we obtain the next corollary.

Collorary 2.3. Let $\left(X,\left\{d_{\alpha}\right\}_{\alpha \in \Lambda}\right)$ be a complete gauge space, and $\phi: X \rightarrow$ $[0, \infty)$ be a lower semi-continuous function. Then for any $x_{0} \in X$, there exists $z \in X$ such that

(i) $\sup _{\alpha} d_{\alpha}\left(x_{0}, z\right) \leq \phi\left(x_{0}\right)-\phi(z)$,

(ii) for each $x \in X$ with $x \neq z$, $\sup _{\alpha} d_{\alpha}(x, z)>\phi(z)-\phi(x)$.

Note that if $\left(X,\left\{d_{\alpha}\right\}_{\alpha \in \Lambda}\right)$ is a complete gauge space and

$$
d(x, y)=\sup _{\alpha \in \Lambda} d_{\alpha}(x, y)<\infty \text { for each } x, y \in X,
$$

then $(X, d)$ is a complete metric space. Hence Corollary 2.3 is a special case of the Ekland's variational principle. However Corollary 2.3 does not require the condition (1).

If $X$ is a complete gauge space endowed with a gauge structure induced by a countable family of pseudo metrics, then by Theorem 2.1, we get the following theorem.

Theorem 2.4. Let $\left(X,\left\{d_{n}\right\}_{n=1}^{\infty}\right)$ be a complete gauge space, and $\left\{\phi_{n}\right\}_{n=1}^{\infty}$ be a family of lower semi-continuous functions from $X$ into $[0, \infty)$. Then for any $x_{0} \in X$, there exists $z \in X$ such that

(i) for each $n \in \mathbb{N}, d_{n}\left(x_{0}, z\right) \leq \phi_{n}\left(x_{0}\right)-\phi_{n}(z)$, 
(ii) for each $x \in X$ with $x \neq z$, there exists $n \in \mathbb{N}$ such that $d_{n}(z, x)>$ $\phi_{n}(z)-\phi_{n}(x)$.

From Theorem 2.4 we have the following corollary.

Collorary $2.5([30])$. Let $(X, \tau)$ be a complete seminormed topological vector space whose topology is generated by a sequence of semi-norms $p_{1} \leq p_{2} \leq \cdots$. Assume that $\phi: X \rightarrow[0, \infty)$ is a lower semicontinuous function. Let $x_{0} \in X$. Then for any $0<\lambda<1$ and any $i \in \mathbb{N}$, there exists $z \in X$ such that

(i) $\lambda p_{i}\left(x_{0}-z\right) \leq \phi\left(x_{0}\right)-\phi(z)$,

(ii) for each $x \in X$ with $x \neq z, \lambda \lim _{n \rightarrow \infty} p_{n}(x-z)>\phi(z)-\phi(x)$.

\section{Fixed point theorems}

From Theorem 2.1 we have the following Caristi-Kirk type fixed point theorem on gauge spaces.

Theorem 3.1. Let $\left(X,\left\{d_{\alpha}\right\}_{\alpha \in \Lambda}\right)$ be a complete gauge space, $\mathcal{F}$ be a countable family of lower semi-continuous functions from $X$ to $[0, \infty)$. Let $\phi: \Lambda \rightarrow \mathcal{F}$ be a map defined by $\phi(\alpha)=\phi_{\alpha}$. If $f: X \rightarrow X$ is a map satisfying for each $x \in X$

$$
d_{\alpha}(x, f x) \leq \phi_{\alpha}(x)-\phi_{\alpha}(f x) \text { for all } \alpha \in \Lambda,
$$

then $f$ has a fixed point in $X$.

Theorem 3.2. Let $\left(X,\left\{d_{\alpha}\right\}_{\alpha \in \Lambda}\right)$ be a complete gauge space, $\mathcal{F}$ be a countable family of lower semi-continuous functions from $X$ to $[0, \infty)$, and let $\phi: \Lambda \rightarrow \mathcal{F}$ be a map defined by $\phi(\alpha)=\phi_{\alpha}$. If $F: X \rightarrow 2^{X}$ is a map satisfying for each $x \in X$, there exists $y \in F x$ such that

$$
d_{\alpha}(x, y) \leq \phi_{\alpha}(x)-\phi_{\alpha}(y) \text { for all } \alpha \in \Lambda,
$$

then $F$ has a fixed point in $X$.

If $\mathcal{F}$ is a singleton, then we have the following result.

Collorary 3.3. Let $\left(X,\left\{d_{\alpha}\right\}_{\alpha \in \Lambda}\right)$ be a complete gauge space, $\phi: X \rightarrow[0, \infty)$ be a lower semi-continuous function. If $f: X \rightarrow X$ is a map satisfying for each $x \in X$,

$$
d_{\alpha}(x, f x) \leq \phi(x)-\phi(f x) \text { for all } \alpha \in \Lambda,
$$

then $f$ has a fixed point in $X$.

Collorary 3.4. Let $\left(X,\left\{d_{\alpha}\right\}_{\alpha \in \Lambda}\right)$ be a complete gauge space, $\phi: X \rightarrow[0, \infty)$ be a lower semi-continuous function. If $F: X \rightarrow 2^{X}$ is a map satisfying for each $x \in X$, there exists $y \in F x$ such that

$$
d_{\alpha}(x, y) \leq \phi(x)-\phi(y) \text { for all } \alpha \in \Lambda,
$$

then $F$ has a fixed point in $X$. 
Collorary $3.5([9])$. Let $X$ be a complete Hausdorff locally convex topological vector space whose topology is generated by a family $\left\{p_{i}\right\}_{i \in I}$ of continuous seminorms, where $I$ is a directed set. Assume that $\phi: X \rightarrow[0, \infty)$ is a lower semicontinuous function and $k: I \rightarrow(0, \infty)$ is a nonincreasing function respect to the ordering in $I$ with $\sup _{i \in I} k(i)<\infty$. If $f: X \rightarrow X$ is a map satisfying for each $x \in X$,

$$
p_{i}(x-f x) \leq k(i)\{\phi(x)-\phi(f x)\} \text { for each } i \in I,
$$

then $f$ has a fixed point in $X$.

Proof. Put $d_{i}(x, y)=\frac{1}{k(i)} p_{i}(x-y)$ for $i \in I$. Then $\left(X,\left\{d_{i}\right\}_{i \in I}\right)$ is a complete gauge space. By Corollary $3.3, f$ has a fixed point in $X$.

In [7], the authors extended the notion of contraction to Hausdorff locally convex linear space $X$ whose topology is generated by a family $\left\{p_{\alpha}\right\}_{\alpha \in \Lambda}$ of semi-norms as follows:

Let $Y \subset X$. We say that $f: Y \rightarrow Y$ is a contraction if, for every $\alpha \in \Lambda$, there exists $k_{\alpha}<1$ such that $p_{\alpha}(f(x)-f(y)) \leq k_{\alpha} p_{\alpha}(x-y)$ for every $x, y \in Y$.

They proved that the Banach contraction principle is still valid for contractions defined on sequentially complete subspace $Y$ of $X$.

We extend the Banach contraction principle to gauge spaces. That is, we obtain the following contraction principle (Theorem 3.6) for a non-self map with inwardness condition on a gauge space with countable pseudo metrics.

Theorem 3.6. Let $\left(X,\left\{d_{n}\right\}_{n=1}^{\infty}\right)$ be a complete gauge space, and $C$ be a nonempty closed subset of $X$. Let $f: C \rightarrow X$ be a map satisfying

(i) for all $n \in \mathbb{N}$, there exists $k_{n} \in(0,1)$ such that $d_{n}(f x, f y) \leq k_{n} d_{n}(x, y)$ for each $x, y \in C$,

(ii) for $x \in C$ with $x \neq f x$, there exists $y \in C$ with $x \neq y$ such that $d_{n}(x, f x)=d_{n}(x, y)+d_{n}(y, f x)$ for all $n \in \mathbb{N}$.

Then $f$ has a fixed point in $C$.

Proof. Assume that $x \neq f x$ for all $x \in C$. Then, for each $x \in X$ there exists $y \in C$ with $x \neq y$ such that $d_{n}(x, f x)=d_{n}(x, y)+d_{n}(y, f x)$ for all $n \in \mathbb{N}$. Then

and so

$$
d_{n}(y, f y)-d_{n}(y, f x) \leq d_{n}(f x, f y) \leq k_{n} d_{n}(x, y)
$$

$d_{n}(y, f y)-d_{n}(x, f x)+d_{n}(x, y) \leq k_{n} d_{n}(x, y)$.

Thus, we have

$$
d_{n}(x, y) \leq \frac{1}{1-k_{n}}\left[d_{n}(x, f x)-d_{n}(y, f y)\right]
$$

By letting $y=g x$ and

$$
\begin{gathered}
\phi_{n}(x)=\frac{1}{1-k_{n}} d_{n}(x, f x) \text { for all } n \in \mathbb{N} \\
d_{n}(x, g x) \leq \phi_{n}(x)-\phi_{n}(g x) .
\end{gathered}
$$


By Theorem 3.1, $g$ has a fixed point in $X$, which is a contradiction. Hence $f$ has a fixed point in $C$.

Collorary 3.7. Let $(X, \tau)$ be a complete topological vector space whose topology is generated by a family $\left\{p_{n}\right\}_{n=1}^{\infty}$ of seminorms and let $C$ be a nonempty closed subset of $X$. Suppose that $f: C \rightarrow X$ is a map satisfying

(i) for all $n \in \mathbb{N}$, there exists $k_{n} \in(0,1)$ such that $p_{n}(f x-f y) \leq k_{n} p_{n}(x-y)$ for all $x, y \in C$,

(ii) $f x \in I_{C}(x)$ for all $x \in C$, where $I_{C}(x)=\{x+\lambda(y-x) \mid y \in C, \lambda \geq 0\}$. Then $f$ has a fixed point in $C$.

\section{Drop theorems}

Daneš [11] proved the following theorem, so called Daneš' drop theorem.

Theorem 4.1 ([11]). Let $X$ be a Banach space. Let $C$ be a closed bounded convex subset of $X$ and let $A$ be a closed subset of $X$. Let $\alpha=\inf \{\|x-y\|$ : $x \in A, y \in C\}>0$. Then there exists $a \in A$ such that $D(a, C) \cap A=\{a\}$.

In $[10,29]$, the authors generalized Daneš' drop theorem to locally convex spaces. We extend this result to semi-normed spaces.

Theorem 4.2. Let $\left(X,\left\{p_{\alpha}\right\}_{\alpha \in \Lambda}\right)$ be a sequentially complete linear topological space whose topology is generated by a family $\left\{p_{\alpha}\right\}_{\alpha \in \Lambda}$ of semi-norms. Let $C$ be a sequentially closed bounded subset of $X$ and let $A$ be a sequentially closed subset of $X$ such that either $C$ or $A$ is a sequentially complete subspace of $X$. If there exists a convex lower semi-continuous map $\phi: X \rightarrow[0, \infty)$ such that $\phi(x)=0$ for all $x \in C$ and $\phi(x) \geq 1$ for all $x \in A$, then for each $x_{0} \in A$, there exists $z \in D\left(x_{0}, C\right)$ such that $D(z, C) \cap A=\{z\}$, where $D\left(x_{0}, C\right)=\operatorname{co}\left(C \cup\left\{x_{0}\right\}\right)$, where co stands for the convex hull operator.

Proof. Let $B=A \cap \overline{D\left(x_{0}, C\right)}$. Then $B$ is a sequentially complete subspace of $X$. Since $\overline{D\left(x_{0}, C\right)}$ is bounded, there exists $r_{\lambda}>0$ such that $\sup \left\{p_{\lambda}(x): x \in\right.$ $\left.\overline{D\left(x_{0}, C\right)}\right\} \leq r_{\lambda}$ for $\lambda \in \Lambda$. By Corollary 2.2, there exists $z \in B$ such that, for each $x \in B$ with $x \neq z$, there exists $\lambda \in \Lambda$ satisfying

$$
p_{\lambda}(x-z)>2 r_{\lambda}(\phi(z)-\phi(x)) .
$$

If there exists $x \in D(z, C) \cap A$ with $x \neq z$, then $x \in B$ and $x=t z+(1-t) c$, where $c \in C$ and $0<t<1$. Then since $\phi$ is convex, we have

$$
\phi(x)=\phi(t z+(1-t) c) \leq t \phi(z)+(1-t) \phi(c) \leq t \phi(z) .
$$

Thus we have

$$
\phi(z) \leq \frac{1}{t} \phi(x)
$$

Since $c, z \in D\left(x_{0}, C\right)$,

$$
p_{\lambda}(x-z)=p_{\lambda}((1-t)(c-z))=(1-t) p_{\lambda}(c-z) \leq 2(1-t) r_{\lambda} .
$$


By (2) and (3)

$p_{\lambda}(x-z)>2 r_{\lambda}(\phi(z)-\phi(x)) \geq 2 r_{\lambda}(\phi(z)-t \phi(z))=2 r_{\lambda}(1-t) \phi(z) \geq 2 r_{\lambda}(1-t)$ which contradicts to (4). Therefore, $D(z, C) \cap A=\{z\}$.

Note that Theorem 4.2 requires the existence of a convex function $\phi$ instead of the convexity of the set $C$. We can prove Theorem 4.1 directly from Theorem 4.2 by putting $\phi(x)=\frac{1}{\alpha} \inf \{\|x-y\|: y \in C\}$. Also, the next corollary show that Theorem 3 in [10] is a special case of Theorem 4.2.

Collorary 4.3 ([10]). Let $(X, \tau)$ be a sequentially complete locally convex space. Let $C$ be a sequentially closed bounded convex set in $X$. For every sequentially closed set $A$, which is strongly Minkowski separated from $C$, there exists $a \in A$ such that $D(a, C) \cap A=\{a\}$.

Proof. Since $A$ and $C$ are strongly Minkowski separated, there exists a Minkowski gauge $p$ on $X$ such that $\alpha=\inf \{p(x-y): x \in A, y \in C\}>0$. For any $x \in X$, define $\phi(x)=\frac{1}{\alpha} \inf \{p(x-y): y \in C\}$. Then $\phi$ is convex and continuous such that $\phi(x)=0$ for $x \in C$ and $\phi(x) \geq 1$ for $x \in A$. By applying Theorem 4.2, we have the desired conclusion.

\section{Palais-Smale condition and coercivity}

Let $X$ be a Banach space. A Gateaux differentiable function $\phi: X \rightarrow \mathbb{R}$ satisfies the Palais-Smale condition if every sequence $\left\{u_{n}\right\}$ in $X$ such that $\left\{\phi\left(u_{n}\right)\right\}$ is bounded and $\left\|\phi^{\prime}\left(u_{n}\right)\right\| \rightarrow 0$ contains a convergent subsequence. A function $\phi: X \rightarrow \mathbb{R}$ is coercive if $\phi(u) \rightarrow \infty$ as $\|u\| \rightarrow \infty$. It is known that for a $C^{1}$ function bounded from below on a Banach space, the Palais-Smale condition implies coercivity (see [8,26]). Caklovic et al. [8] proved the following theorem by using the Ekeland's variational principle.

Theorem 5.1. Let $X$ be a Banach space and let $\phi: X \rightarrow \mathbb{R}$ be a Gateaux differentiable lower semi-continuous function satisfying the Palais-Smale condition. If $\phi$ is bounded from below, then $\phi$ is coercive.

We generalize this theorem to a complete locally convex topological vector space with extended notions of the Palais-Smale condition and coercivity. Let $X$ be a locally convex topological vector space generated by a family $\left\{p_{\lambda}\right\}_{\lambda \in \Lambda}$ of seminorms. Recall that, a function $\phi: X \rightarrow \mathbb{R}$ is Gateaux differentiable at $x_{0}$ if there exists a continuous linear map $\phi^{\prime}\left(x_{0}\right): X \rightarrow \mathbb{R}$, such that for any $u \in X$

$$
\lim _{t \rightarrow 0^{+}} \frac{\phi\left(x_{0}+t u\right)-\phi\left(x_{0}\right)}{t}=\phi^{\prime}\left(x_{0}\right)(u) .
$$

Assume $p(x)=\sup _{\lambda \in \Lambda} p_{\lambda}(x)<\infty$ for each $x \in X$. Let $X^{*}=\{\Gamma: X \rightarrow \mathbb{R} \mid \Gamma$ is continuous and linear $\}$. For $\Gamma \in X^{*}$, define

$$
|\Gamma|=\sup _{x \neq 0} \frac{|\Gamma(x)|}{p(x)}=\sup _{p(x)=1}|\Gamma(x)| .
$$


Then we know $|\Gamma|<\infty$ for $\Gamma \in X^{*}$. We define the Palais-Smale condition on a locally convex topological vector space as follows:

A Gateaux differentiable function $\phi: X \rightarrow \mathbb{R}$ satisfies the Palais-Smale condition if every sequence $\left\{u_{n}\right\}$ in $X$ such that $\left\{\phi\left(u_{n}\right)\right\}$ is bounded and $\left|\phi^{\prime}\left(u_{n}\right)\right| \rightarrow$ 0 contains a convergent subsequence. A function $\phi:\left(X,\left\{p_{\lambda}\right\}_{\lambda \in \Lambda}\right) \rightarrow \mathbb{R}$ is coercive if for each $\lambda \in \Lambda, \phi(u) \rightarrow \infty$ as $p_{\lambda}(u) \rightarrow \infty$.

Theorem 5.2. Let $(X, \tau)$ be a complete locally convex topological vector space generated by a family $\left\{p_{\lambda}\right\}_{\lambda \in \Lambda}$ of seminorms, and let $\phi: X \rightarrow \mathbb{R}$ be a Gateaux differentiable lower semicontinuous function satisfying the Palais-Smale condition. If $\phi$ is lower bounded, then $\phi$ is coercive.

Proof. On the contrary, suppose that $\phi$ is not coercive. Then there exists a $\lambda_{0} \in \Lambda$ such that $\underline{\lim }_{p_{\lambda_{0}(u)} \rightarrow \infty} \phi(u)=c \in \mathbb{R}$. Thus, for each $n \in \mathbb{N}$, there exists $u_{n} \in X$ with $p_{\lambda_{0}}\left(u_{n}\right) \geq 2 n$ and $\phi\left(u_{n}\right) \leq c+\frac{1}{n}$. By Corollary 2.2, for every $n \in \mathbb{N}$, there exists $v_{n} \in X$ such that for any $\lambda \in \Lambda$

$$
\frac{n c+1}{n^{2}} p_{\lambda}\left(u_{n}-v_{n}\right) \leq \phi\left(u_{n}\right)-\phi\left(v_{n}\right)
$$

and for each $u \neq v_{n}$, there exists $\alpha \in \Lambda$ such that

$$
\frac{n c+1}{n^{2}} p_{\alpha}\left(u-v_{n}\right)>\phi\left(v_{n}\right)-\phi(u) \text {. }
$$

Thus we have

$$
\phi\left(v_{n}\right) \leq \phi\left(u_{n}\right) \leq c+\frac{1}{n} \text { for each } n=1,2, \ldots
$$

Therefore, $\left\{\phi\left(v_{n}\right)\right\}$ is bounded. With (5) and (6), we have $p_{\lambda_{0}}\left(u_{n}-v_{n}\right) \leq n$. Since $p_{\lambda_{0}}\left(u_{n}\right) \geq 2 n$,

$$
p_{\lambda_{0}}\left(v_{n}\right) \geq p_{\lambda_{0}}\left(u_{n}\right)-p_{\lambda_{0}}\left(u_{n}-v_{n}\right) \geq 2 n-n \geq n .
$$

Therefore, $\left\{v_{n}\right\}$ has no convergent subsequence.

Let $u \in X$ with $u \neq 0$. Then there exists $\alpha \in \Lambda$ such that for $t>0$,

$$
\frac{n c+1}{n^{2}}>\frac{\phi\left(v_{n}\right)-\phi\left(v_{n}+t u\right)}{p_{\alpha}\left(v_{n}+t u-v_{n}\right)}=\frac{\phi\left(v_{n}\right)-\phi\left(v_{n}+t u\right)}{t p_{\alpha}(u)} .
$$

Thus we have

$$
\frac{\phi\left(v_{n}\right)-\phi\left(v_{n}+t u\right)}{t}<\frac{n c+1}{n^{2}} p_{\alpha}(u) \leq \frac{n c+1}{n^{2}} p(u) .
$$

So

$$
-\phi^{\prime}\left(v_{n}\right)(u)=\lim _{t \rightarrow 0^{+}} \frac{\phi\left(v_{n}\right)-\phi\left(v_{n}+t u\right)}{t} \leq \frac{n c+1}{n^{2}} p(u) .
$$

Also,

$$
-\phi^{\prime}\left(v_{n}\right)(-u) \leq \frac{n c+1}{n^{2}} p(-u)
$$


Therefore

$$
\left|\phi^{\prime}\left(v_{n}\right)\right|=\sup _{p(u) \neq 0} \frac{\left|\phi^{\prime}\left(v_{n}\right)(u)\right|}{p(u)} \leq \frac{n c+1}{n^{2}} \rightarrow 0 \text { as } n \rightarrow \infty .
$$

This shows that $\phi$ does not satisfy Palais-Smale condition, a contradiction.

Acknowledgment. The authors would like to thank the referee for helpful comments regarding this paper. In particular, the authors thank the referee for providing references [31] and [32].

\section{References}

[1] R. P. Agarwal and D. O.'Regan, Fixed -point theorems for multivalued maps with closed values on complete gauge spaces, Appl. Math. Lett. 14 (2001), no. 7, 831-836.

[2] S. Al-Homidan, Q. H. Ansari, and J. C. Yao, Some generalizations of Ekeland-type variational principle with applications to equilibrium problems and fixed point theory, Nonlinear Anal. 69 (2008), no. 1, 126-139.

[3] J. P. Aubin and H. Frankowska, Set-Valued Analysis, Birkhäuser, Boston, Berlin, 1990.

[4] M. Bianchi, G. Kassay, and R. Pini, Existence of equilibria via Ekeland's principle, J. Math. Anal. Appl. 305 (2005), no. 2, 502-512.

[5] H. Brezis and F. E. Browder, A general principle on ordered sets in nonlinear functional analysis, Advances in Math. 21 (1976), no. 3, 355-364.

[6] F. E. Browder, Normal solvability and the Fredholm alternative for mappings into infinite dimensional manifolds, J. Funct. Anal. 8 (1971), 250-274.

[7] G. L. Cain Jr and M. Z. Nashed, Fixed points and stability for a sum of two operators in locally convex spaces, Pacific J. Math. 39 (1971), 581-592.

[8] L. Caklovic, S. Li, and M. Willem, A note on Palais-Smale condition and coercivity, Differential Integral Equations 3 (1990), no. 4, 799-800.

[9] F. Cammaroto, A. Chinni, and G. Sturiale, A remark on Ekeland's principle in locally convex topological vector spaces, Mathematical and Computer Modeling 30 (1990), 7579.

[10] L. Cheng, Y. Zhou, and F. Zhang, Danes' drop theorem in locally convex spaces, Proc. Amer. Math. Soc. 124 (1996), no. 12, 3699-3702.

[11] J. Daněs, A geometric theorem useful in nonlinear functional analysis, Boll. Un. Mat. Ital. (4) 6 (1972), 369-372.

[12] Equivalence of some geometric and related results of nonlinear functional analysis, Comment. Math. Univ. Carolin. 26 (1985), no. 3, 443-454.

[13] D. G. De Figueiredo, The Ekeland Variational Principle with Applications and Detours, Tata Institue of Fundamental esearch, Bombay, 1989.

[14] S. Dolecki and J. P. Penot, The Clark's tangent cone and limits of tangent cones, Publ. Math. Pau 2 (1983), 1-11.

[15] J. Dugundji, Topology, Ally and Bacon, Boston, 1966.

[16] I. Ekeland, Sur les problémes variationnels, C. R. Acad. Sci. Paris Sér. A-B 275 (1972), 1057-1059.

[17] _ On the variational principle, J. Math. Anal. Appl. 47 (1974), 324-353.

[18] _ On convex minimization problems, Bull. Amer. Math. Soc. 1 no. 3 (1979), 445-474.

[19] R. Espínola and W. A. Kirk, Set-valued contractions and fixed points, Nonlinear Anal. 54 (2003), no. 3, 485-494.

[20] J. X. Fang and X. Y. Lin, Fixed point theorems for set-valued $\Phi$-generalized contractions on gauge spaces, Nonlinear Anal. 69 (2008), no. 1, 201-207. 
[21] M. Frigon, Fixed point results for generalized contractions in gauge spaces and application, Proc. Amer. Math. Soc. 128 (2000), no. 10, 2957-2965.

[22] J. R. Giles and D. N. Kutzarova, Characterisation of drop and weak drop properties for closed bounded convex sets, Bull. Austral. Math. Soc. 43 (1991), no. 3, 377-385.

[23] J. R. Giles, B. Sims, and A. C. Yorke, On the drop and weak drop properties for a Banach space, Bull. Austral. Math. Soc. 41 (1990), no. 3, 503-507.

[24] A. Göpfert, Chr. Tammer, H. Riahi, and C. Zălinescu, Variational Method in Partially Ordered Spaces, Springer-Verlag, New York, Berlin, Heidelberg, 2003.

[25] D. N. Kutzarova, On drop property of convex sets in Banach space, in "Constructive Theory of Functions '87, Sofa", 283-287.

[26] S. Li, An existence theorem on multiple critical points and its application in nonlinear PDE, Acta Mathematica Scientica 4 (1984).

[27] J. P. Penot, The drop theorem, the petal theorem and Ekeland's variational principle, Nonlinear Anal. 10 (1986), no. 9, 813-822.

[28] R. R. Phelps, Convex Functions, Monotone operators and Differentiability, SpringerVerlag, New York, Berlin, Heidelberg-New York, 1989.

[29] J. Qiu, Local completeness and Drop theorem, J. Math. Anal. Appl. 266 (2002), no. 2, $288-297$.

[30] _ A version of Ekeland's variational principle in countable semi-normed spaces, J. Math. Res. Exposition 24 (2004), no. 1, 1-6.

[31] J. H. Qiu and S. Rolewicz, Local completeness of locally pseudoconvex spaces and Borwein-Preiss variational principle, Studia Math. 183 (2007), no. 2, 99-115.

[32] _ Ekeland's variational principle in locally p-convex spaces and related results, Studia Math. 186 (2008), no. 3, 219-235.

[33] S. Rolewicz, On drop property, Studia Math. 85 (1987), 27-35.

[34] W. Takahashi, Nonlinear Functional Analysis, Yokohama Publishers, Yokohama, Japan, 2000.

[35] M. Turinici, Mapping theorems via variable drops in Banach spaces, Istit. Lombardo Accad. Sci. Lett. Rend. A 114 (1980), 164-168.

[36] X. Y. Zheng, A drop theorem in topological linear spaces, Chinese Ann. Math. Ser. A 21 (2000), no. 2, 141-148.

\author{
JONG-SOOK BAE \\ Department of Mathematics \\ MOYNGJI UNIVERSITY \\ Yongin 449-728, KoreA \\ E-mail address: jsbae@mju.ac.kr \\ Seong-Hoon Cho \\ Department of Mathematics \\ Hanseo University \\ Chungnam 356-706, Korea \\ E-mail address: shcho@hanseo.ac.kr \\ JEONG-JIN KIM \\ Department of Mathematics \\ MOYNGJI UNIVERSITY \\ Yongin 449-728, KoreA \\ E-mail address: jjkim@mju.ac.kr
}

